\title{
COVID-19: Association Between Increase of Behavioral and Psychological Symptoms of Dementia During Lockdown and Caregivers' Poor Mental Health
}

\author{
Elodie Pongan ${ }^{\mathrm{a}, \mathrm{b}, 1, *}$, Jean-Michel Dorey ${ }^{\mathrm{c}, 1}$, Céline Borg $^{\mathrm{a}}$, Jean Claude Getenet ${ }^{\mathrm{a}}$, Romain Bachelet $^{\mathrm{b}}$, \\ Charles Lourioux ${ }^{\mathrm{c}}$, Bernard Laurent ${ }^{\mathrm{a}}$, COVCARE Group ${ }^{2}$, Romain Rey ${ }^{\mathrm{c}}$ and Isabelle Rouch ${ }^{\mathrm{a}, \mathrm{b}, \mathrm{d}}$ \\ ${ }^{a}$ Neurology Unit, CM2R, CHU de Saint Etienne, Hôpital Nord, Saint Etienne, France \\ ${ }^{\mathrm{b}}$ Clinical and Research Memory Center of Lyon, Hospices civils de Lyon, Hôpital des Charpennes, Villeurbanne, \\ France \\ ${ }^{\mathrm{c}}$ Psychiatry Unit, Centre hospitalier le Vinatier, Bron, France \\ d INSERM, U1219, Bordeaux Population Health Center, University of Bordeaux, Bordeaux, France
}

Accepted 10 February 2021

Pre-press 23 February 2021

\begin{abstract}
.
Background: From March 2020, the support and care systems for caregivers and people with dementia (PWD) were suspended or dramatically changed due to the lockdown during the world pandemic of COVID-19. Thus, these changes in living conditions have had deleterious consequences on the behavior of PWD and subsequently on their caregivers' mental health, the two being linked.

Objective: Our study aimed to examine changes in behavior among PWD and to look for associations between the evolution of behavioral and psychological symptoms of dementia (BPSD) and caregivers' mental health in the context of COVID-19. Methods: The study was conducted among caregivers of PWD living at home in France. Caregivers were interviewed via an anonymous cross-sectional online survey during the first lockdown between April 15 and June 15, 2020.

Results: Three hundred and eighty-nine caregivers accompanying a relative living at home participated in the study; $43.3 \%$ of the PWD presented a worsening of BPSD during the lockdown. With multivariate logistic regressions, a significant association was observed between "more BPSD" and burden, anxiety and depression, between "BPSD equivalent" and anxiety and depression, and between "emerging BPSD" and only depression.

Conclusion: The lockdown seems to have an impact on behavioral disorders in PWD and these disorders are associated with poorer mental health of caregivers. Our findings suggest attention should be given to caregivers of PWD who have BPSD before lockdown and the need for continued consultations and professional help in case of new lockdowns.
\end{abstract}

Keywords: Behavioral disorder, caregivers, COVID-19, lockdown, mental health

\footnotetext{
${ }^{1}$ These authors contributed equally to this work.

${ }^{2}$ Mathieu Herrmann, Céline Borg, Laurence Cadet, Jean-Michel Dorey, Stéphanie Dirson, Céline Dubien, Jean Claude Getenet, Cécile Lebrun Givois, Charles Lourioux, Elodie Pongan, Romain Rey, Isabelle Rouch, Françoise Vincent Grangette

*Correspondence to: Elodie Pongan, MSc, Centre Mémoire de Ressources et de Recherche, Neurology unit, University Hospital of Saint-Etienne, 42055 Saint Etienne, France. Tel.: + 3347712 73 97; E-mail: elodie.pongan@chu-lyon.fr.
}

\section{INTRODUCTION}

In addition to cognitive impairment, the behavioral and psychological symptoms of dementia (BPSD) are a major aspect of neurodegenerative diseases. Indeed, they contribute to patients' loss of autonomy and are associated with poorer outcomes. Symptoms depend on the specific dysfunctional brain systems and on patient's related factors (i.e., premorbid personal- 
ity, personal history, and current socio-environmental context) [1-3]. Not only do BPSD have a significant impact on patient well-being, but they also cause stress and suffering for their relatives. Caring for people with dementia (PWD) can lead to the development of psychiatric disorders such as depression $[4,5]$. Therefore, decreasing the caregivers' burden is essential [6] to improve their mental health and to reduce the patients behavioral disorders, the two being linked [7].

From March 2020, the support and care systems for caregivers and patients were suspended or dramatically changed due to the world pandemic of the new coronavirus disease, COVID-19. This pandemic forced authorities to enact drastic lockdown measures, prohibiting travel except for compelling reasons and on the sole condition of being provided with a derogatory certificate [8]. As a consequence, all non-urgent medical consultations were postponed. Professional caregivers, nursing aids, and home meal deliveries were rearranged or removed. Rehabilitation activities such as physiotherapy or speech therapy were stopped at least temporarily. In addition, patients living at home were no longer allowed to visit day-centers. Moreover, following corona virus guidance (such as social distancing) or limiting walks is particularly difficult for PWD to respect because they usually have a strong need for physical activity. Thus, it is likely that these changes in living conditions have had deleterious consequences on the behavior of PWD and subsequently on the mental health of their caregivers, since many of them had to compensate for the missing aids and spent the lockdown with their sick relative.

Few studies measured the effect of such adverse socio-environmental changes on behavioral disorders in patients with PWD and their impact on their caregivers. After 5 weeks of lockdown, Lara et al. [9] observed a worsening of the mean of neuropsychiatric symptoms in patients with Alzheimer's disease (AD) or mild cognitive impairment while no difference in caregivers' and patients' quality of life was found. Boutoleau-Bretonnière et al. [10] showed that neuropsychiatric changes observed during the lockdown were restricted to a minority of patients with AD. Patients with neuropsychiatric changes had lower cognitive functioning compared with patients who did not demonstrate neuropsychiatric changes. However, those results were obtained on a small sample of patients. Moreover, mental health assessment was restricted to the subset of caregivers whose relative exhibited a change of BPSD during lockdown. More recently, results from a large Italian cohort showed BPSD worsening among almost $60 \%$ of patients and stress-related feelings experienced by two-thirds of caregivers [11]. Nevertheless, caregivers' mental health was not assessed by standardized scales and association with BPSD was not studied.

The objective of the present study was 1) to describe the prevalence of behavioral changes in PWD during the COVID-19 lockdown and 2) to look for associations between such behavioral changes and various aspects of caregivers' mental health, especially burden, depression, and anxiety.

\section{METHODS}

\section{Study population}

The study was conducted among caregivers of PWD living at home in France. The participants were recruited with a snowball sampling method from various sources, e-mails sent to members of the France Alzheimer association, memory centers, via social networks or information given during memory center video appointments, or visits carried out in the month following the end of the lockdown. A minimum age of 18 years old was required.

\section{Data collection}

We conducted an anonymous cross-sectional online survey between April 15 and June 15, 2020. A hard copy of the survey, along with a stamped envelope, was provided to people without a web access who asked for it. The online questionnaire directly implemented an Excel database. Data from the paper versions were manually entered by a clinical research associate into the Excel database.

\section{Survey}

The Checklist for Reporting Results of Internet E-Surveys (CHERRIES) has been used for the methodology [12]. The questionnaire was divided into two parts. The first one mostly consisted of closed questions to collect socio-demographic and medical data. The second one included standardized scales to assess the caregivers' mental health. Approximatively thirty minutes were necessary to complete the questionnaire.

\section{Socio-demographic and medical data}

Data on the PWD, their caregiver, and the caregiver's network before and after lockdown were collected. Changes in the support provided by care- 
givers to their relative since the beginning of the lockdown were also recorded. More precisely, the question of the evolution of the PWD's behavioral disorders was addressed by the following request: Since lockdown, has your relative presented psychological or behavioral manifestations?

The participant had to choose between 'No' and 'Yes'. If 'Yes', they were asked to clarify whether the BPSD were: 1) 'not present before lockdown', 2) 'present before lockdown but not more disruptive', 3)'more disruptive than before lockdown'. They were then asked to specify the type of BPSD of their relative (anxiety, depression, irritability/aggression, insomnia, hallucination, delusions, apathy).

\section{Caregivers mental health assessment}

Four scales were used to evaluate the caregiver's mental health.

The GAD-7 scale (The General Anxiety Disorder7) [13] consists of 7 items and measures the presence and severity of anxiety symptoms, specifically linked to the $D S M-I V$ criteria. Each of the 7 items is scored from 0 to 3 . The total score ranges from 0 to 21 . A score of 5 to 9 can be interpreted as mild anxiety, 10 to 14 as moderate anxiety, and 15 to 21 as severe anxiety. A score below 5 means no anxiety. A cut off of 10 is recommended for the identification of generalized anxiety cases [13].

The CES-D scale (Center for Epidemiologic Studies- Depression) [14] contains 20 items and assesses the subject's mood by evaluating the experienced symptoms or behaviors associated with depression. The frequency of symptom onset during the past week is measured using a 4-point Likert scale. The highest score is 60 points. A cut-off of 20 is recommended to identify significant depressive symptoms [15].

The ZBI (Zarit Burden Interview [16]) is a widelyused self-report measure of caregiver burden. The Mini Zarit (French short version of ZBI) was used in this study [17]. It consists of 7 questions to which the caregivers answered "never" (0 point), "sometimes" (0.5 point), or "nearly always" (1 point). The total score ranges from 0 (no burden) to 7 (higher burden). A score above 5.5 is considered as a severe burden.

The level of self-reported stress was assessed with a visual analog scale ranging from 0 to 10 . Caregivers had to answer the following question: Since the beginning of the lockdown, on a scale from 0 (complete absence of stress) to 10 (maximum imaginable stress) what is your stress level? This scale was constructed specifically for the purpose of this study.

\section{Statistical analyses}

Descriptive characteristics were compared between these groups. ANOVA was used for quantitative variables (e.g., age or symptoms duration). Chi Square $\left(\chi^{2}\right)$ test was used for qualitative variables (e.g., gender or education).

Four groups of PWD were defined based on the evolution of their BPSD during lockdown: "no BPSD" group (i.e., absence of behavioral and psychological symptoms of dementia), "emerging BPSD" (i.e., emergence of behavioral and psychological symptoms of dementia during lockdown), "BPSD equivalent" (i.e., behavioral and psychological symptoms of dementia present prior to lockdown and remaining present without increase), and "more BPSD” (i.e., behavioral and psychological symptoms of dementia present before lockdown and increased during the lockdown).

Caregivers' mental health scores were compared between these groups with ANOVA.

For each of the mental health scales a cut-off score was used to separate participants with poor mental health (PMH) from those with good mental health $(\mathrm{GMH})$. A cut off of 5.5 was used for the mini Zarit, corresponding to severe burden [17]. A cut off of 10 was used for the GAD 7. Indeed, a score of 10 or greater on the GAD-7 represents, according to the authors, a reasonable cut point for identifying cases of GAD [13]. A cut off of 20 was used for the CESD, meaning significant depressive symptoms [15], and a score superior or equal to 6 was considered as 'clinically significant stress' for self-reported stress scale. For each of the mental health scales, multivariate logistic regression analyzes adjusted for the sex and age of the caregiver, living condition (with or without the patient) and patient behavioral symptoms duration were performed. Caregivers' mental health scores were used as outcome and BPSD groups as independent variables.

A $p$ value $<0.05$ was considered to be statistically significant (no corrections made for multiple comparisons). All statistical analyses were performed using SPSS version 21 (SPSS Software, Chicago, IL).

\section{RESULTS}

\section{Participants' characteristics}

Three hundred and eighty-nine caregivers accompanying a relative living at home participated in the study. Caregivers and patients' characteristics 
Table 1

Participants' characteristics according to BPSD evolution

\begin{tabular}{|c|c|c|c|c|c|c|}
\hline & $\begin{array}{c}\text { Total } \\
n=383\end{array}$ & $\begin{array}{c}\text { No BPSD } \\
n=55\end{array}$ & $\begin{array}{c}\text { Emerging } \\
\text { BPSD } \\
n=23\end{array}$ & $\begin{array}{c}\text { BPSD } \\
\text { equivalent } \\
n=162\end{array}$ & $\begin{array}{c}\text { More } \\
\text { BPSD } \\
n=143\end{array}$ & $P$ \\
\hline \multicolumn{7}{|l|}{ Caregivers } \\
\hline Age, $\mathrm{y}$, mean $\pm \mathrm{SD}$ & $62.8 \pm 13$ & $66.33 \pm 13.7$ & $62.3 \pm 9.9$ & $63.8 \pm 12.8$ & $60.5 \pm 13.1$ & 0.025 \\
\hline Gender female, n (\%) & $277(72.3)$ & $34(61.8)$ & 17 (73.9) & $115(71)$ & $111(77.6)$ & 0.12 \\
\hline Link with patient, $\mathrm{n}(\%)$ & & & & & & 0.4 \\
\hline Spouse & $207(54.6)$ & $34(64.2)$ & $13(56.5)$ & $90(55.6)$ & $70(49.6)$ & \\
\hline Children & $150(39.6)$ & $16(30.2)$ & $10(43.5)$ & $61(37.7)$ & $63(44.7)$ & \\
\hline Grandchildren & $5(1.3)$ & $1(1.9)$ & $0(0)$ & $1(0.6)$ & $3(2.1)$ & \\
\hline Brother/sister & $2(0.5)$ & $1(1.9)$ & $0(0)$ & $0(0)$ & $1(0.7)$ & \\
\hline Others & $15(4)$ & $1(1.9)$ & $0(0)$ & $10(6.2)$ & $4(2.8)$ & \\
\hline Living with patient, n (\%) & $255(66.6)$ & $38(69.1)$ & $15(65.2)$ & $108(66.7)$ & $94(65.7)$ & 0.9 \\
\hline \multicolumn{7}{|l|}{ Patients } \\
\hline Age, $y$, mean $\pm S D$ & $76.9 \pm 8.7$ & $75.9 \pm 9$ & $76.6 \pm 8.5$ & $77.3 \pm 9$ & $76.9 \pm 8.2$ & 0.76 \\
\hline Gender female, $\mathrm{n}(\%)$ & $203(53)$ & $33(60)$ & $12(52.2)$ & $84(51.9)$ & $74(51.7)$ & 0.77 \\
\hline Symptom duration, $y$, mean \pm SD & $6 \pm 4.1$ & $5.8 \pm 4.3$ & $5.5 \pm 5$ & $6 \pm 4.3$ & $6.2 \pm 3.7$ & 0.9 \\
\hline Diagnosis etiology, $\mathrm{n}(\%)$ & & & & & & 0.45 \\
\hline $\mathrm{AD}$ & $243(67.5)$ & $29(56.9)$ & $14(63.6)$ & $101(66.4)$ & $99(73.3)$ & \\
\hline FTD & $27(7.5)$ & $3(5.9)$ & $1(4.5)$ & $13(8.6)$ & $10(7.4)$ & \\
\hline LBD & $23(6.4)$ & $4(7.8)$ & $2(9.1)$ & $9(5.9)$ & $8(5.9)$ & \\
\hline ORD & $44(12.2)$ & $9(17.6)$ & $5(22.7)$ & $18(11.8)$ & $12(8.9)$ & \\
\hline DNK & $23(6.4)$ & $6(11.8)$ & $0(0)$ & $11(7.2)$ & $6(4.4)$ & \\
\hline Hospitalization for BPSD, $\mathrm{n}(\%)$ & $84(21.9)$ & $9(16.4)$ & $4(17.4)$ & $41(25.3)$ & $30(21)$ & \\
\hline Cessation of personal care assistant (\%) & $60(15.7)$ & $3(5.5)$ & $5(21.7)$ & $26(16)$ & $26(18.2)$ & 0.27 \\
\hline Cessation of day care centers $(\%)$ & $125(32.6)$ & $8(14.5)$ & $15(45.2)$ & $42(25.9)$ & $60(42)$ & $<0.0001$ \\
\hline
\end{tabular}

No BPSD, no behavioral and psychological symptoms of dementia; Emerging BPSD, emergence of behavioral and psychological symptoms of dementia during lockdown; BPSD equivalent, behavioral and psychological symptoms of dementia were present before lockdown and remain present without increase; More BPSD, behavioral and psychological symptoms of dementia were present before lockdown and increased; AD, Alzheimer disease; LBD, Lewy body disease; FTD, frontotemporal dementia; ORD, other related disorders (other dementia, Parkinson's disease, stroke); DNK, caregiver does not know diagnosis.

were compared according to BPSD evolution during lockdown; 383 caregivers provided information about this evolution (Table 1). Regarding the evolution of BPSD, a total of 143 PWD (37.3\%) had "more BPSD", 162 (42.3\%) had "equivalent BPSD", $23(6 \%)$ had "emerging BPSD", and 55 (14.4\%) had "no BPSD". Only the caregiver's age was significantly different $(p=0.025)$ between groups. No significant difference was found for all other socio-demographic and medical characteristics. The diagnoses reported in the 337 patients for whom the caregiver knew and provided the information are $\mathrm{AD}(n=243)$, frontotemporal dementia (FTD) $(n=27)$, Lewy bodies dementia (LBD) $(n=23)$, other dementias ( $n=44$, including 16 vascular dementia, 5 Parkinson's dementia, and 23 other related diseases). Figure 1 provides the percentage of PWD according to each BPSD. Apathy was reported in 185 patients (48.1\%), anxiety in 173 patients (44.7\%), aggression, irritability in 165 patients $(42.6 \%)$, depression in 90 patients $(23.3 \%)$, insomnia in 84 patients $(21.7 \%)$, hallucinations in 71 patients $(18.3 \%)$, and delusion in 62 patients $(16 \%)$.

\section{Caregivers' mental health according to BPSD changes}

All caregivers' mental health scores, Mini Zarit, CES-D, GAD 7, and self-reported stress differed significantly between the four groups. The lowest scores were obtained by the "no BPSD" group and the highest scores by the "more BPSD" group. These results are provided in Table 2. Multivariate analyses adjusted for caregivers' age and sex, the fact of living with patient, and symptom duration are provided in Table 3. For each caregivers' mental health scale, a significant association was observed between PMH and "more BPSD" group compared to "no BPSD" group. The same associations were found among the "BPSD equivalent" except for the Mini-Zarit scale. Finally, a significant association was shown among the "emerging BPSD" for CES-D scores only.

\section{DISCUSSION}

Our study was the first to assess the mental health of PWD's caregivers during lockdown with 


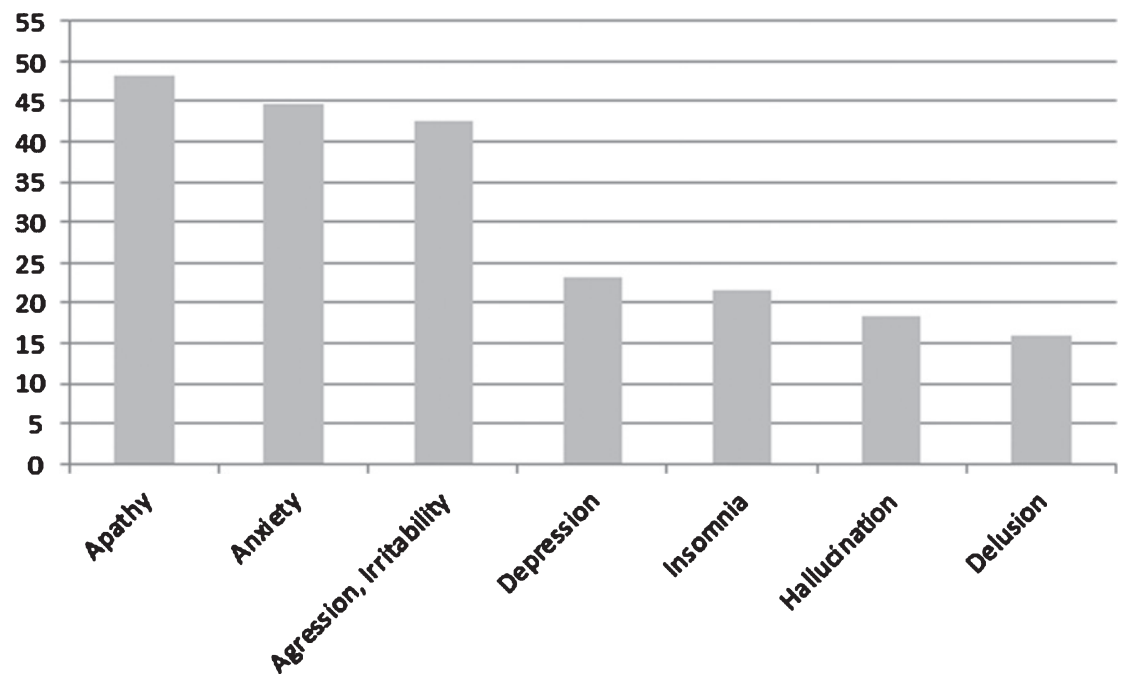

Fig. 1. Percentage of patients according to each BPSD.

Table 2

Scores of caregivers on mental health scales according to BPSD evolution

\begin{tabular}{|c|c|c|c|c|c|c|}
\hline & $\begin{array}{c}\text { Total } \\
n=383\end{array}$ & $\begin{array}{c}\text { No BPSD } \\
n=55\end{array}$ & $\begin{array}{c}\text { Emerging } \\
\text { BPSD } \\
n=23\end{array}$ & $\begin{array}{c}\text { BPSD } \\
\text { equivalent } \\
n=162\end{array}$ & $\begin{array}{c}\text { More } \\
\text { BPSD } \\
n=143\end{array}$ & $P$ \\
\hline Mini Zarit mean \pm SD & $4.29 \pm 1.68$ & $3.11 \pm 1.75$ & $3.93 \pm 1.38$ & $4.04 \pm 1.61$ & $5.11 \pm 1.36$ & $<0.0001$ \\
\hline $\mathrm{GAD} 7$ mean $\pm \mathrm{SD}$ & $9.3 \pm 5.8$ & $6.61 \pm 6.12$ & $8.82 \pm 5.6$ & $8.47 \pm 5.57$ & $11.35 \pm 5.6$ & $<0.0001$ \\
\hline CES-D mean \pm SD & $22.01 \pm 11.54$ & $17.95 \pm 10.86$ & $22.32 \pm 12$ & $20.96 \pm 10.82$ & $24.57 \pm 12.01$ & 0.012 \\
\hline Self-reported stress mean \pm SD & $5.29 \pm 2.65$ & $3.75 \pm 2.5$ & $4.87 \pm 2.75$ & $5.02 \pm 2.55$ & $6.24 \pm 2.45$ & $<0.0001$ \\
\hline
\end{tabular}

No BPSD, no behavioral and psychological symptoms of dementia; Emerging BPSD, emergence of behavioral and psychological symptoms of dementia during lockdown; BPSD equivalent, behavioral and psychological symptoms of dementia were present before lockdown and remain present without increase; More BPSD, behavioral and psychological symptoms of dementia were present before lockdown and increase.

Table 3

Association between caregivers scores on mental health scales and BPSD evolution-multivariate logistic regression models

\begin{tabular}{|c|c|c|c|c|c|c|c|c|}
\hline & \multicolumn{2}{|c|}{$\begin{array}{c}\text { Mini Zarit } \\
(\geq 5.5, \mathrm{PMH} \text { versus GMH) }\end{array}$} & \multicolumn{2}{|c|}{$\begin{array}{c}\text { GAD } 7 \\
(\geq 10, \text { PMH versus GMH })\end{array}$} & \multicolumn{2}{|c|}{$\begin{array}{c}\text { CES-D } \\
(\geq 20, \text { PMH versus GMH) }\end{array}$} & \multicolumn{2}{|c|}{$\begin{array}{c}\text { Self-reported stress } \\
(\geq 6, \mathrm{PMH} \text { versus GMH) }\end{array}$} \\
\hline & $\mathrm{OR}(\mathrm{CI})$ & $p$ & $\mathrm{OR}(\mathrm{CI})$ & $p$ & $\mathrm{OR}(\mathrm{CI})$ & $p$ & $\mathrm{OR}(\mathrm{CI})$ & $p$ \\
\hline No BPSD & 1 & - & 1 & - & 1 & - & 1 & - \\
\hline $\begin{array}{l}\text { Emerging } \\
\text { BPSD }\end{array}$ & $1.003(0.26-3.87)$ & 0.99 & $3.33(1.03-10.79)$ & 0.05 & $3.63(1.16-11.41)$ & 0.03 & $2.24(0.757-6.68)$ & 0.15 \\
\hline $\begin{array}{l}\text { BPSD } \\
\text { equivalent }\end{array}$ & $1.52(0.63-3.65)$ & 0.35 & $2.85(1.21-6.74)$ & 0.02 & $2.82(1.29-6.17)$ & 0.01 & $2.29(1.07-4.88)$ & 0.03 \\
\hline More BPSD & $4.14(1.75-9.79)$ & 0.001 & $6.07(2.56-14.41)$ & $<0.0001$ & $4.16(1.87-9.27)$ & $<0.0001$ & $3.93(1.83-8.46)$ & $<0.0001$ \\
\hline
\end{tabular}

Multivariate logistic regressions adjusted on caregivers' age and sex, living conditions (living or not with PWD) and symptom duration. No BPSD, no behavioral and psychological symptoms of dementia; Emerging BPSD, emergence of behavioral and psychological symptoms of dementia during lockdown; BPSD equivalent, behavioral and psychological symptoms of dementia were present before lockdown and remain present without increase; More BPSD, behavioral and psychological symptoms of dementia were present before lockdown and increased; $\mathrm{PMH}$, poor mental health; GMH, good mental health.

standardized scales on a large cohort. We aimed to examine changes in behavior among PWD and to look for associations between the evolution of BPSD and caregivers' mental health.
We reported that almost half of the patients exhibited an onset or worsening of BPSD during the limited period of lockdown. Additionally, our study highlighted an association between increased BPSD 
during lockdown and caregivers' poor mental health evaluated by scales assessing anxiety, general stress, depression and burden.

\section{Prevalence of BPSD changes}

BPSD are common manifestations of major cognitive disorders but their progression is supposed to be fluctuating and slow over the course of AD [18]. In a cohort also including other etiologies, as in our study (FTD, LBD, vascular dementia, and other dementia), the evolution of NPI score over a year on average was not significant (17.55 versus 18.50) [19].

In the present study, sudden worsening or newonset of BPSD have been reported in almost 50\% of the PWD over a short period of 2 months following the setting-up of quarantine. This peak of challenging behaviors is consistent with other studies conducted during lockdown that revealed an increase of neuropsychiatric symptoms in 25 to $60 \%$ of people suffering from AD or related disorders [9-11].

Many factors influencing the emergence of BPSD have been identified, among them environmental stressors play a crucial role [20]. We suspect that the disruption of day-to-day habits and routines due to lockdown could have generated distress in PWD impaired to cope with unusual situations because of their cognitive deficits. The rising of BPSD may be related to the drastic limitation of social and physical activities that are recommended to prevent such symptoms [21]. Additionally, the closure of respite facilities and the restricted access to expert memory centers have prevented caregivers from getting advices and psychological support to face the difficulties encountered. It is worthy to note that the prevalence of aggression/irritability in our study is higher than described in the literature [3, 22, 23]. These results come from studies including only AD patients. This can explain the differences observed. We can also hypothesize that a prolonged proximity between the caregiver and his relative can promote tensions and mirror reactions. Eventually, the lockdown could also have impacted caregivers' perceptions of their relative's BPSD with a subjective impression of increased BPSD, only because more time together was spent.

According to our results, the observed evolution of BPSD during lockdown is not explained by socio-demographic variables such as the patients' age or sex. Remarkably, the evolution of BPSD was neither linked to medical variables such as dementia duration or etiology nor by the presence of previous hospitalizations for BPSD. These results differ from those of the Italian cohort [11] showing that BPSD types varied according to dementia etiology and patients' gender. Interestingly, the age of the caregiver was related to the course of BPSD: the older the caregiver is, the less BPSD he reports regarding his relative. One possible explanation is that the youngest caregivers are the children of PWD living distantly, whereas the oldest are the spouses living in the same household. These latter situations could be associated with a better knowledge leading to a greater tolerance toward BPSD. Another explanation is that older caregivers support older patients who exhibit less severe BPSD as compared to younger PWD. However, none of these three variables were related to the presence of behavioral disorders. More qualitative information is needed to address this issue.

Finally, the cessation of day care center activities during lockdown was also associated with the evolution of BPSD. They may have increased because of this cessation, but also because patients resorting to day care centers are probably the most severe ones.

\section{BPSD changes and caregivers' mental health}

Most studies assessing the impact of BPSD on caregivers' mental health focused on their burden or distress. In the present study, we reported associations between evolution of BPSD and caregivers' burden, depression, anxiety status, and self-reported stress. It should be highlighted that the significance of the observed associations persisted even after adjusting for various well-described potential confounders (i.e., caregivers' sex [5], living or not with PWD, and symptom duration $[24,25])$.

First, we found an association between increased BPSD during lockdown and the presence of a severe burden in caregivers. Our results are consistent with previous studies. Berger et al. observed a significant link between PWDs' behavioral disorders and the burden of their caregivers [26]. Dauphinot et al. reported an association between BPSD and caregiver burden regardless of etiology [19]. Other studies also reported a significant association between BPSD and high levels of caregiver distress [27-31]. During the COVID-19 crisis, Boutoleau-Bretonnière et al. also showed an association between increased BPSD and caregivers' burden during lockdown for AD patients but not for behavioral variant of FTD in which the sole presence of BPSD impacts the burden [32].

In the present study, only BPSD worsening during lockdown were associated with a severe burden. 
Surprisingly, such an association was not observed when BPSD were absent before lockdown. This may be due to the fact that emerging BPSD were less severe than worsening ones or they may have not lasted for long enough to burden the caregivers. Unfortunately, we did not assess BPSD severity in the present study. Thus, we could not look for an association between caregivers' burden and BPSD severity, although such an association has been previously reported. In the Dauphinot et al. study, a worsening of BPSD symptoms over time (increase of $\geq 4$ points in the NPI) contributed to an increase in caregiver burden [19]. Similarly, using a cross-sectional design, Mukherjee et al. observed that caregiver distress worsened with increasing number and magnitude of BPSD [33]. Altogether these results suggest that caregivers' burden is related to BPSD increase and severity.

Caregivers' PMH could also be linked to the cessation of respite structures such as day care centers. Indeed, the "more BSPD" patients are more resorting to these centers.

Secondly, we report an association between the presence of significant depressive symptoms and the presence of BPSD, regardless of the anteriority of the BPSD (i.e., whether they were present or not before lockdown), and regardless of their evolution (i.e., whether they worsened or not during lockdown). This is consistent with a previous study reporting an association between caregivers' depression and disturbances in sleep-activity rhythm in PWD [26]. However, in this latter study, no association between depression and other BPSD was observed. Since the lockdown situation has been previously associated with higher incidence of depressive symptoms [34, 35]; it may be suggested that our results reflect the deleterious effect of both BPSD and lockdown situation on caregivers' mood.

Finally, we found a significant association between the presence of BPSD prior to lockdown and caregivers' self-reported stress as well as anxiety status. Similarly to burden, the onset of BPSD during lockdown was not associated with self-reported stress or anxiety status. It may thus be suggested that caregivers supporting relatives with long-lasting BPSD are characterized by a reduced coping ability to the deleterious effects of lockdown as compared to other caregivers. In line with this hypothesis, in the present study, the strongest associations were observed between increased BPSD and burden $(\mathrm{OR}=4.14)$, depressive symptoms $(\mathrm{OR}=4.16)$, anxiety $(\mathrm{OR}=6.07)$, and stress $(\mathrm{OR}=3.93)$. Alternatively, emerging
BPSD may be less severe than worsening ones More studies assessing the severity level of BPSD are needed to confirm the association between BPSD severity and caregivers' stress or anxiety status. Overall, our results suggest that caregivers supporting relatives with long-lasting BPSD may be considered as a vulnerable subgroup among caregivers.

\section{Strengths and limitations}

The present study has several strengths. Firstly, all the previous studies exploring the deleterious consequences of lockdown on mental health focused on general population $[36,37]$ or PWD themselves [38]. To our knowledge, the present study is the first to assess caregivers' mental health (except the burden) during the lockdown. Secondly, while previous studies assessing the impact of BPSD on caregivers' mental health solely focused on their burden or stress, our study has explored the potential consequences of BPSD on specific mental health symptoms such as depression or anxiety. In this regard, the use of validated scales and clinical cutoffs gives clinical significance to our results. Finally, the inclusion of a large number of potential confounding factors in the multivariate analyses (caregivers' sex, living or not with PWD, and symptom duration) may be underscored as another strength.

The findings of this study have to be seen in light of some limitations. Firstly, some limitations are inherent to questionnaire surveys: 1) only caregivers interested and motivated by the subject of the study responded, which reduces the representativeness of our sample; 2) some participants may have had problems understanding the questions asked. For instance, in the present study, the rate of depression in PWD evaluated by caregivers was smaller than the rate previously reported [3]. This discrepancy may reflect the difficulty for caregivers to differentiate apathy from depression.

Secondly, no direct measure of PWD cognitive status, such as Mini-Mental State Examination score, was available in our study. However, the symptoms duration was used as proxy to take into account dementia severity. Thirdly, BPSD evolution was subjectively assessed by caregivers which is an important limit. A standardized assessment performed by a clinician would have allowed to evaluate the severity of BPSD. However, such an assessment was not possible during the lockdown.

Another limitation is that the power to detect differences in the "emerging BPSD" group is low as 
there are only 23 people in that group. This is why the confidence intervals in the logistic regression are wide and might be the reason why there is only an association between "emerging BPSD" and depression and not the other outcomes when using logistic regression.

Lastly, it would have been interesting to explore BPSD evolution and consequences on caregivers' mental health after lockdown. However, the survey methodology (anonymous online questionnaire, cross-sectional design) did not allow us to collect these data.

\section{CONCLUSION}

Our study shows that the specific context of lockdown, which deprived people of non-emergency outings and consultations, exerted deleterious consequences both on PWD and their caregivers. Regarding PWD, a worsening of BPSD was reported in $43.3 \%$ of cases over a short period of 2 months. Regarding caregivers, we report associations between increased BPSD during lockdown and severe burden, clinically significant depression, anxiety status, and stress. Notably, our results suggest that caregivers supporting relatives with long-lasting BPSD may be considered as a more vulnerable subgroup among caregivers.

We are currently facing an upsurge in COVID-19 cases and can assume that this situation will last for several months before a vaccine is available for everyone. New lockdowns, at least local ones, are likely to be enacted. Our results emphasize the importance of maintaining support and resources to help caregivers accompanying their relatives during lockdown. In this regard, phone- or video-consultations should be encouraged and home care services (e.g., professional caregivers, home meal deliveries) maintained as long as possible. Finally, specific attention should be paid to caregivers supporting relatives with longlasting BPSD since they may be characterized by a reduced coping ability to the deleterious effects of lockdown as compared to other caregivers.

\section{ACKNOWLEDGMENTS}

The France Alzheimer association and psychologists from the Memory Center from St Etienne university hospital (neurology unit) contributed to the dissemination of the online survey. There is no sponsor to report.
Authors' disclosures available online (https:// www.j-alz.com/manuscript-disclosures/20-1396r2).

\section{REFERENCES}

[1] Apostolova LG, Di LJ, Duffy EL, Brook J, Elashoff D, Tseng C-H, Fairbanks L, Cummings JL (2014) Risk factors for behavioral abnormalities in mild cognitive impairment and mild Alzheimer's disease. Dement Geriatr Cogn Disord 37, 315-326.

[2] Burgio LD, Butler FR, Roth DL, Hardin JM, Hsu C-C, Ung K (2000) Agitation in nursing home residents: The role of gender and social context. Int Psychogeriatr 12, 495-511.

[3] Zhao Q-F, Tan L, Wang H-F, Jiang T, Tan M-S, Tan L, Xu W, Li J-Q, Wang J, Lai T-J, Yu J-T (2016) The prevalence of neuropsychiatric symptoms in Alzheimer's disease: Systematic review and meta-analysis. J Affect Disord 190, 264-271.

[4] Liu S, Li C, Shi Z, Wang X, Zhou Y, Liu S, Liu J, Yu T, Ji Y (2017) Caregiver burden and prevalence of depression, anxiety and sleep disturbances in Alzheimer's disease caregivers in China. J Clin Nurs 26, 1291-1300.

[5] Mahoney R, Regan C, Katona C, Livingston G (2005) Anxiety and depression in family caregivers of people with Alzheimer disease: The LASER-AD Study. Am J Geriatr Psychiatry 13, 795-801.

[6] Moniz Cook ED, Swift K, James I, Malouf R, De Vugt M, Verhey F (2012) Functional analysis-based interventions for challenging behaviour in dementia. Cochrane Database Syst Rev, CD006929.

[7] Isik AT, Soysal P, Solmi M, Veronese N (2019) Bidirectional relationship between caregiver burden and neuropsychiatric symptoms in patients with Alzheimer's disease: A narrative review. Int J Geriatr Psychiatry 34, 1326-1334.

[8] Attestation numérique de déplacement dérogatoire. https:// media.interieur.gouv.fr/attestation-couvre-feu-covid-19/

[9] Lara B, Carnes A, Dakterzada F, Benitez I, Piñol-Ripoll G (2020) Neuropsychiatric symptoms and quality of life in Spanish patients with Alzheimer's disease during the COVID-19 lockdown. Eur J Neurol 27, 1744-1747.

[10] Boutoleau-Bretonnière C, Pouclet-Courtemanche H, Gillet A, Bernard A, Deruet AL, Gouraud I, Mazoue A, Lamy E, Rocher L, Kapogiannis D, El Haj M (2020) The effects of confinement on neuropsychiatric symptoms in Alzheimer's disease during the COVID-19 crisis. J Alzheimers Dis 76, 41-47.

[11] Cagnin A, Di Lorenzo R, Marra C, Bonanni L, Cupidi C, Laganà V, Rubino E, Vacca A, Provero P, Isella V, Vanacore N, Agosta F, Appollonio I, Caffarra P, Pettenuzzo I, Sambati R, Quaranta D, Guglielmi V, Logroscino G, Filippi M, Tedeschi G, Ferrarese C, Rainero I, Bruni AC, SINdem COVID-19 Study Group (2020) Behavioral and psychological effects of coronavirus disease-19 quarantine in patients with dementia. Front Psychiatry 11, 578015.

[12] Eysenbach G (2004) Improving the quality of web surveys: The Checklist for Reporting Results of Internet E-Surveys (CHERRIES). J Med Internet Res 6 , e34.

[13] Spitzer RL, Kroenke K, Williams JBW, Löwe B (2006) A brief measure for assessing generalized anxiety disorder: The GAD-7. Arch Intern Med 166, 1092.

[14] Verdier-Taillefer M-H, Gourlet V, Fuhrer R, Alpérovitch A (2001) Psychometric properties of the center for epidemiologic studies-depression scale in multiple sclerosis. Neuroepidemiology 20, 262-267. 
[15] Vilagut G, Forero CG, Barbaglia G, Alonso J (2016) Screening for depression in the general population with the Center for Epidemiologic Studies Depression (CES-D): A systematic review with meta-analysis. PLoS One 11, e0155431.

[16] Zarit SH, Reever KE, Bach-Peterson J (1980) Relatives of the impaired elderly: Correlates of feelings of burden. Gerontologist 20, 649-655.

[17] Revel Da Rocha V, Haritchabalet I, Kervinio C, Drode M, Sauvanier M, Geny C, Placines B, Roux C, Albert $\mathrm{D}$, Delafournière F, Dartigues JF, Rocha-Rego V, Albert DB (2002) Construction d'une échelle simplifiée pour la détection en médecine générale du fardeau de l'aidant d'une personne âgée dépendante. L'année Gérontologique 16, 131-137.

[18] the COOPERA Study Group, Fernández M, Gobartt AL, Balañá M (2010) Behavioural symptoms in patients with Alzheimer's disease and their association with cognitive impairment. BMC Neurol 10, 87.

[19] Dauphinot V, Ravier A, Novais T, Delphin-Combe F, Mouchoux C, Krolak-Salmon P (2016) Risk factors of caregiver burden evolution, for patients with subjective cognitive decline or neurocognitive disorders: A longitudinal analysis. J Am Med Dir Assoc 17, 1037-1043.

[20] Dorey J-M, Herrmann M, Schuster J, Von Guten A, Lepetit A (2019) Stratégie thérapeutiques des troubles psychocomportementaux dans les troubles neurocognitifs de la personne âgée. doi : 10.1016/S0246-1072(19)81456-6

[21] Cankurtaran ES (2014) Management of behavioral and psychological symptoms of dementia. Noro Psikiyatri Arsivi 51, 303-312.

[22] Benoit M, Staccini P, Brocker P, Benhamidat T, Bertogliati C, Lechowski L, Tortrat D, Robert PH (2003) [Behavioral and psychologic symptoms in Alzheimer's disease: Results of the REAL.FR study]. Rev Med Interne 24(Suppl 3), 319s-324s.

[23] Benoit M, Robert PH, Staccini P, Brocker P, Guerin O, Lechowski L, Vellas B, REAL.FR Group (2005) One-year longitudinal evaluation of neuropsychiatric symptoms in Alzheimer's disease. The REAL.FR Study. J Nutr Health Aging 9, 95-99.

[24] Black CM, Ritchie CW, Khandker RK, Wood R, Jones E, Hu X, Ambegaonkar BM (2018) Non-professional caregiver burden is associated with the severity of patients' cognitive impairment. PLoS One 13, e0204110.

[25] Mioshi E, Foxe D, Leslie F, Savage S, Hsieh S, Miller L, Hodges JR, Piguet O (2013) The impact of dementia severity on caregiver burden in frontotemporal dementia and Alzheimer disease. Alzheimer Dis Assoc Disord 27, 68-73.

[26] Berger G, Bernhardt T, Weimer E, Peters J, Kratzsch T, Frolich L (2005) Longitudinal study on the relationship between symptomatology of dementia and levels of subjective burden and depression among family caregivers in memory clinic patients. J Geriatr Psychiatry Neurol 18, 119-128.

[27] Hiyoshi-Taniguchi K, Becker CB, Kinoshita A (2018) What behavioral and psychological symptoms of dementia affect caregiver burnout? Clin Gerontol 41, 249-254.

[28] Huang S-S, Lee M-C, Liao Y-C, Wang W-F, Lai T-J (2012) Caregiver burden associated with behavioral and psychological symptoms of dementia (BPSD) in Taiwanese elderly. Arch Gerontol Geriatr 55, 55-59.

[29] Rosdinom R, Zarina MZN, Zanariah MS, Marhani M, Suzaily W (2013) Behavioural and psychological symptoms of dementia, cognitive impairment and caregiver burden in patients with dementia. Prev Med 57, S67-S69.

[30] Shaji K, George R, Prince M, Jacob K (2009) Behavioral symptoms and caregiver burden in dementia. Indian J Psychiatry 51, 45.

[31] Song J-A, Oh Y (2015) The association between the burden on formal caregivers and behavioral and psychological symptoms of dementia (BPSD) in Korean elderly in nursing homes. Arch Psychiatr Nurs 29, 346-354.

[32] Boutoleau-Bretonnière $\mathrm{C}$, Pouclet-Courtemanche $\mathrm{H}$, Gillet A, Bernard A, Deruet A-L, Gouraud I, Lamy E, Mazoué A, Rocher L, Bretonnière C, El Haj M (2020) Impact of confinement on the burden of caregivers of patients with the behavioral variant of frontotemporal dementia and Alzheimer disease during the COVID-19 crisis in France. Dement Geriatr Cogn Disord Extra 10, 127-134.

[33] Mukherjee A, Biswas A, Roy A, Biswas S, Gangopadhyay G, Das SK (2017) Behavioural and psychological symptoms of dementia: Correlates and impact on caregiver distress. Dement Geriatr Cogn Disord Extra 7, 354-365.

[34] Majumdar P, Biswas A, Sahu S (2020) COVID-19 pandemic and lockdown: Cause of sleep disruption, depression, somatic pain, and increased screen exposure of office workers and students of India. Chronobiol Int 37, 1191-1200.

[35] Tang F, Liang J, Zhang H, Kelifa MM, He Q, Wang P (2021) COVID-19 related depression and anxiety among quarantined respondents. Psychol Health 36, 164-178.

[36] Ahmed MZ, Ahmed O, Aibao Z, Hanbin S, Siyu L, Ahmad A (2020) Epidemic of COVID-19 in China and associated psychological problems. Asian J Psychiatry 51, 102092.

[37] Wang C, Pan R, Wan X, Tan Y, Xu L, Ho CS, Ho RC (2020) Immediate psychological responses and associated factors during the initial stage of the 2019 coronavirus disease (COVID-19) epidemic among the general population in China. Int J Environ Res Public Health 17, 1729.

[38] El Haj M, Altintas E, Chapelet G, Kapogiannis D, Gallouj K (2020) High depression and anxiety in people with Alzheimer's disease living in retirement homes during the covid-19 crisis. Psychiatry Res 291, 113294. 